\title{
Atividade física e qualidade de vida em indivíduos renais crônicos
}

\section{Physical activity and quality of life in chronic kidney individuals}

\author{
Luana Cecconello' 1 (D) \\ Edinara Moraes Morais ${ }^{2}$ (1) \\ Karen Rafaela Okaseski Scopel ${ }^{3}$ (1)
}
Eniva Miladi Fernandes Stumm ${ }^{4}$ (1)
Paulo Ricardo Moreira ${ }^{5}$ (1) Eliane Roseli Winkelmann ${ }^{6}$ (1)

1-4Universidade Regional do Noroeste do Estado do Rio Grande do Sul (ljui). Rio Grande do Sul, Brasil. luanacecconello@hotmail.com, edinara.morais@iffarroupilha.edu.br, karen_scopel@hotmail.com, eniva@unijui.edu.br

5Universidade de Cruz Alta (Cruz Alta), Rio Grande do Sul, Brasil. prm.paulomoreira@gmail.com ${ }^{6}$ Autora para correspondência. Universidade Regional do Noroeste do Estado do Rio Grande do Sul (ljui). Rio Grande do Sul, Brasil. elianew@unijui.edu.br

RESUMO | INTRODUÇÃO: As alterações impostas pela doença renal e por seu tratamento interferem negativamente na qualidade de vida dos indivíduos com doença renal crônica. A atividade física é relatada como uma alternativa terapêutica nesta população, porém a maioria dos pacientes submetidos à hemodiálise apresentam baixos níveis de atividade física. OBJETIVO: verificar a correlação entre a atividade física e qualidade de vida em indivíduos com doença renal crônica em hemodiálise. MÉTODOS: Este estudo é observacional, analítico, descritivo e quantitativo, desenvolvido em uma das Unidades de Hemodiálise da região Noroeste do Rio Grande do Sul, Brasil, na atenção clínica terciária, durante o período de novembro de 2018 a fevereiro de 2019. Foram incluídos indivíduos maiores de 18 anos e em tratamento hemodialítico por doença renal crônica há mais de três meses, pertencentes ao serviço de hemodiálise. Os critérios de exclusão foram os indivíduos com diagnóstico de doença renal aguda; aqueles que apresentaram aparentemente dificuldades em compreender, responder ou que não realizaram completamente os instrumentos de avaliação propostos (qualidade de vida e pedômetros), indivíduos que no momento da avaliação não apresentaram condições clínicas estáveis. A coleta de dados foi realizada pela análise dos prontuários clínicos e eletrônicos e entrevista semiestruturada. Utilizou-se avaliação pelos pedômetros e pelo questionário Kidney Disease and Quality of Life Short-Form-KDQOL-SFIM. Análises de modelagem por regressão foram realizadas para testar a associação entre o número de passos/dia e os desfechos avaliados RESULTADOS: Foram incluídos na amostra 40 pacientes, destes, $70 \%$ são homens, com média de idade de 59,9 $\pm 13,0$ anos. Na correlação entre atividade física e qualidade de vida, o número de passos/dia teve correlação significativa com as dimensões sintomas e problemas $(r=0,523 ; p=0,003)$, efeitos da doença $(r=0,458 ; p=0,010)$, função sexual $(r=0,361 ; p=0,050)$, sono $(r=0,357 ; p=0,049)$, função física $(r=0,617 ; p=<0,001)$, papel físico $(r=0,504 ; p=0,004)$, dor $(r=0,496 ; p=0,005)$, bem estar emocional $(r=0,407 ; p=0,023)$, papel emocional $(r=0,435 ; p=0,014)$, função social $(r=0,522 ; p=0,003)$, energia/fadiga $(r=0,436 ; p=0,014)$ e composição física $(r=0,598 ; p=<0,001)$. As variáveis idade, índice de massa corporal, tempo de hemodiálise e sexo não apresentaram correlação com o número de passos/dia. CONCLUSÃO: Houve correlação positiva entre atividade física e qualidade de vida, ou seja, quanto maior a média de número de passos/dia melhor a qualidade de vida de indivíduos em hemodiálise.
ABSTRACT | INTRODUCTION: The changes imposed by kidney disease and its treatment negatively affect the quality of life of individuals with kidney disease. Physical activity is reported as a therapeutic alternative in this population; however, most patients undergoing hemodialysis have low levels of physical activity. OBJECTIVE: To verify the correlation between physical activity and quality of life in individuals with chronic kidney disease undergoing hemodialysis. METHODS: This study is observational, analytical, descriptive, and quantitative developed in one of the Hemodialysis Units in the Northwest region of Rio Grande do Sul, Brazil, in tertiary clinical care, during the period from November 2018 to February 2019. Individuals over 18 years old and undergoing hemodialysis for chronic kidney disease for more than three months; belonging to the hemodialysis service. Data collection was performed by analyzing clinical and electronic medical records and semi-structured interviews. Evaluation using pedometers and the Kidney Disease and Quality of Life Short-FormKDQOL-SFTM questionnaire was used. Regression modeling analyzes were performed to test the association between the number of steps/ day and the evaluated outcomes. RESULTS: 40 (Forty) patients were included in the sample, of which $70 \%$ are men, with a mean age of 59.9 \pm 13.0 years. In the correlation between physical activity and quality of life, the number of steps / day had a significant correlation with the dimensions symptoms and problems $(r=0,523 ; p=0,003)$, effects of the disease $(r=0,458 ; p=0,010)$, sexual function $(r=0,361 ; p=0,050)$, sleep $(r=0,357 ; p=0,049)$, physical function $(r=0,617 ; p=<0,001)$, physical role $(r=0,504 ; p=0,004)$, pain $(r=0,496 ; p=0,005)$, emotional well-being $(r=0,407 ; p=0,023)$, emotional role $(r=0,435 ; p=0,014)$, social function $(r=0,522 ; p=0,003)$, energy / fatigue $(r=0,436 ; p=0,014)$ and physical composition $(r=0,598 ; p=<0,001)$. The sample profile variables did not correlate with the number of steps/day. CONCLUSION: There was a positive correlation between physical activity and quality of life; that is, the higher the average number of steps/day, the better the quality of life of individuals undergoing hemodialysis.

KEYWORDS: Chronic Kidney Failure. Hemodialysis. Physical activity. Health level. Disease Impact Profile.
PALAVRAS-CHAVE: Insuficiência Renal Crônica. Hemodiálise. Atividade física. Nível de Saúde. Perfil de Impacto da Doença. 


\section{Introdução}

A Doença Renal Crônica (DRC), progressiva e irreversível, tem assumido destaque como um grande impacto em saúde pública ${ }^{1}$. Os dados dos últimos censos brasileiros são preocupantes e demonstram que os números referentes à doença renal continuam crescendo, o que a torna uma doença reconhecida como complexa e exigente de intervenções abrangentes e múltiplas². As alterações impostas no organismo, pela doença e pelo próprio tratamento, interferem negativamente em todos os aspectos da qualidade de vida (QV) e atingem de uma forma tão homogenia o biopsicossocial do indivíduo e, secundariamente, o seu contexto 3 .

Os pacientes em tratamento dialítico podem apresentar limitações físicas, psicológicas e sociais que afetam seu estilo de vida, além das alterações adicionais que Ihes são impostas pela clínica da doença renal, o que tem impacto expressivo na multidimensionalidade do conceito de qualidade de vida relacionada a saúde ${ }^{4}$.

A atividade física é relatada como uma alternativa terapêutica não farmacológica e de baixo custo ${ }^{5}$ além de beneficiar a função cardiorrespiratória, melhorar a qualidade de vida ${ }^{6}$ e ser preditora de desfechos clínicos adversos em pacientes com doença renall ${ }^{7}$. Contudo, a maioria dos pacientes submetidos à hemodiálise apre-

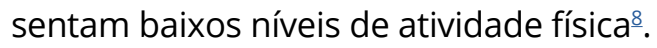

Em contrapartida, mudanças comportamentais refletem positivamente na amenização do quadro. Se as interações entre o indivíduo, equipe de saúde e contexto seguirem hábitos, comportamentos e ações saudáveis, os doentes renais tendem a ter melhor sobrevida e serão menos vulneráveis aos efeitos deletérios das complicações impostas pela doença e pelo próprio tratamento. Neste sentido, a hipótese do presente estudo é que um estilo de vida mais ativo está relacionado com melhor qualidade de vida em doentes renais crônicos em tratamento hemodialítico. Desta forma, o objetivo do estudo foi verificar a correlação entre a atividade física e qualidade de vida, além de correlacionar o número de passos/dia com as variáveis de perfil da amostra (idade, índice de massa corporal, tempo de hemodiálise e sexo) em pacientes com doença renal em tratamento hemodialítico.
Esta pesquisa se caracteriza por um estudo observacional, analítico, descritivo e quantitativo, aprovado pelo Comitê de Ética em Pesquisa com Seres Humanos da Universidade Regional do Noroeste do Estado do Rio Grande do Sul (UNIJUí), seguindo as diretrizes propostas na resolução 466/2012 do Conselho Nacional de Saúde sobre pesquisas com seres humanos com parecer $n^{\circ} 3.012 .096$ e CAAE 90954218.6.0000.5350.

O estudo foi desenvolvido em uma das Unidades de Hemodiálise da região Noroeste do Estado do Rio Grande do Sul, Brasil, que atende um total de aproximadamente 160 indivíduos (agudos e crônicos). A pesquisa foi realizada durante o período de novembro de 2018 a fevereiro de 2019. Foram considerados critérios de inclusão para esta pesquisa ser maior de 18 anos; indivíduos em tratamento hemodialítico por doença renal crônica há mais de três meses; pertencentes ao serviço de hemodiálise. Os critérios de exclusão foram os indivíduos com diagnóstico de doença renal aguda; indivíduos que apresentaram aparentemente dificuldades em compreender, responder ou que não realizaram completamente os instrumentos de avaliação propostos (qualidade de vida e pedômetros), indivíduos que no momento da avaliação não apresentaram condições clínicas estáveis.

Para amostragem da pesquisa, foi realizada a análise de todos os pacientes de um único centro de hemodiálise. Após aplicar os critérios de inclusão e exclusão, 40 pacientes compreenderam a amostra do estudo (Figura 1). A coleta de dados foi realizada por fisioterapeutas, e a equipe realizou um treinamento prévio para a adequação e padronização na coleta de dados. Por meio da análise dos prontuários clínicos e eletrônicos, foi realizada uma entrevista semiestruturada elaborada para este estudo, a fim de se obter o perfil da amostra, questionário de qualidade de vida e avaliação do nível de atividade física de acordo com a descrição abaixo.

As variáveis obtidas para o perfil da amostra foram idade, sexo, índice de massa corporal (IMC), tempo de hemodiálise, etiologia da doença renal, tipo de acesso, e realização ou não de acompanhamento pré-dialítico. 
Foi mensurado o peso $(\mathrm{kg})$ utilizando uma balança portátil, estatura $(\mathrm{cm}) \mathrm{com}$ um estadiômetro portátil e a partir deste realizado o cálculo do IMC (IMC = peso/ altura ${ }^{2}$ ). Para avaliação da qualidade de vida utilizou-se o Kidney Disease and Quality of Life Short-Form (KDQOL$\mathrm{SF}^{\mathrm{TM}}$ ), questionário específico, validado no Brasil, e que contêm dimensões importantes para a avaliação da qualidade de vida de indivíduos renais crônicos. Este foi aplicado durante a sessão de hemodiálise e é composto por 36 itens (8 domínios) do 36-Item Short Form Health Survey (SF-36), e mais 43 itens específicos (11 domínios) relacionados a DRC dialítica. Contém uma avaliação de saúde geral. O KDQOL-SFTM é autoaplicável e o escore final varia de 0 a 100, sendo que valores mais próximos ao 100 correspondem a uma melhor qualidade de vida relacionada à saúde?

O nível de atividade física foi verificado pela quantificação do número de passos, em que foi utilizado pedômetros, dispositivos de contagem de passos sensíveis à detecção de movimento corporal, e, para a classificação, utilizou-se o sugerido por Tudor-Locke ${ }^{10,11}$. Os indivíduos receberam um pedômetro (Omron Healthcare, modelo HJA-310, Kyoto - Japão) e a orientação de utilizá-lo preso à cintura, continuamente, exceto no banho e/ou atividades que molhassem/danificassem o equipamento, e no período do sono noturno. $O$ equipamento foi entregue em um dia de sessão de hemodiálise e recolhido na próxima sessão. Foi realizada uma média de número de passos diários, uma vez que a cada 24 horas o dispositivo registrava e reiniciava a quantificação. Não foram utilizados ponto de corte.

As variáveis categóricas foram apresentadas por frequências e porcentagens e as numéricas como médias e desvios-padrão (média \pm DP) ou mediana (intervalo interquartil), conforme o teste de Shapiro-Wilk. Foi utilizado a mediana (IQ) para as variáveis tempo de hemodiálise e para as demais foi utilizado a média $\pm D P$. Análises de modelagem por regressão foram realizadas para testar a associação entre o número de passos/dia e os desfechos avaliados. Para avaliar a correlação entre o nível de atividade física e as dimensões da qualidade de vida utilizou-se o teste de correlação de Spermann. Considerou-se um nível de significância de $5 \%$. Os dados coletados foram agrupados, tabulados e analisados no software Statistical Package for the Social Sciences (SPSS Inc., Chicago, IL, EUA) versão 23.0.

\section{Resultados}

No momento da coleta de dados, o serviço atendia aproximadamente 160 indivíduos, foram excluídos 42 indivíduos: 10 (óbitos), 06 (transferências de unidade), 02 (altas), 06 (tratamento intensivo), 18 (não aceitaram participar do estudo). Foram incluídos 118 indivíduos, destes 78 responderam os questionários e 53 aceitaram utilizar os pedômetros. Para responder nosso objetivo de estudo era necessário participar adequadamente de todos os métodos de avaliação, logo, um total de 40 pacientes com DRC em tratamento hemodialítico foram incluídos na amostra (Figura 1).

A Tabela 1 exibe as características demográficas e clínicas da amostra. A média de idade foi de 59,9 \pm 13,0 anos; $70 \%$ eram homens. A mediana do tempo de hemodiálise foi de 34,5 meses. A etiologia da DRC mais predominante foi a HAS associada a DM (32,5\%), seguido da HAS (30\%). A mediana do número de passos/dia foi de $2690(918,0$ - 5009,0) passos, (Figura 2).

De acordo com a regressão linear, o número de passos/dia teve correlação significativa com as seguintes dimensões do KDQOL-SFTM: sintomas e problemas $(r=0,523 ; p=0,003)$, efeitos da doença $(r=0,458 ; p=0,010)$, função sexual $(r=0,361 ; p=0,050)$, sono $(0,357 ; p=0,049)$, função física $(r=0,617 ; p<0,001)$, papel físico $(r=0,504 ; p=0,004)$, dor $(r=0,496 ; p=0,005)$, bem estar emocional $(r=0,407 ; p=0,023)$, papel emocional $(r=0,435 ; p=0,014)$, função social $(r=0,522 ; p=0,003)$, energia/fadiga $(r=0,436 ; p=0,014)$, composição física $(r=0,598 ; p<0,001)$. Estes dados e demais dimensões encontram-se na Tabela 2.

Também, foi realizada análise de regressão para número de passos/dia e variáveis de perfil da amostra que não apresentaram correlação significativa (idade, IMC, tempo de hemodiálise, sexo feminino, escore saúde geral - KDQOL-SFTM), Tabela 3.

Os escores de cada dimensão do questionário de QV encontram-se na Tabela 4. Os menores escores de QV foram identificados nas dimensões situação de trabalho, função sexual e limitações físicas, enquanto os maiores escores foram obtidos nas dimensões função cognitiva, apoio social e encorajamento da equipe de saúde. 
Figura 1. Seleção dos pacientes submetidos á hemodiálise das Unidades de Hemodiálise da região Noroeste .2018-2019

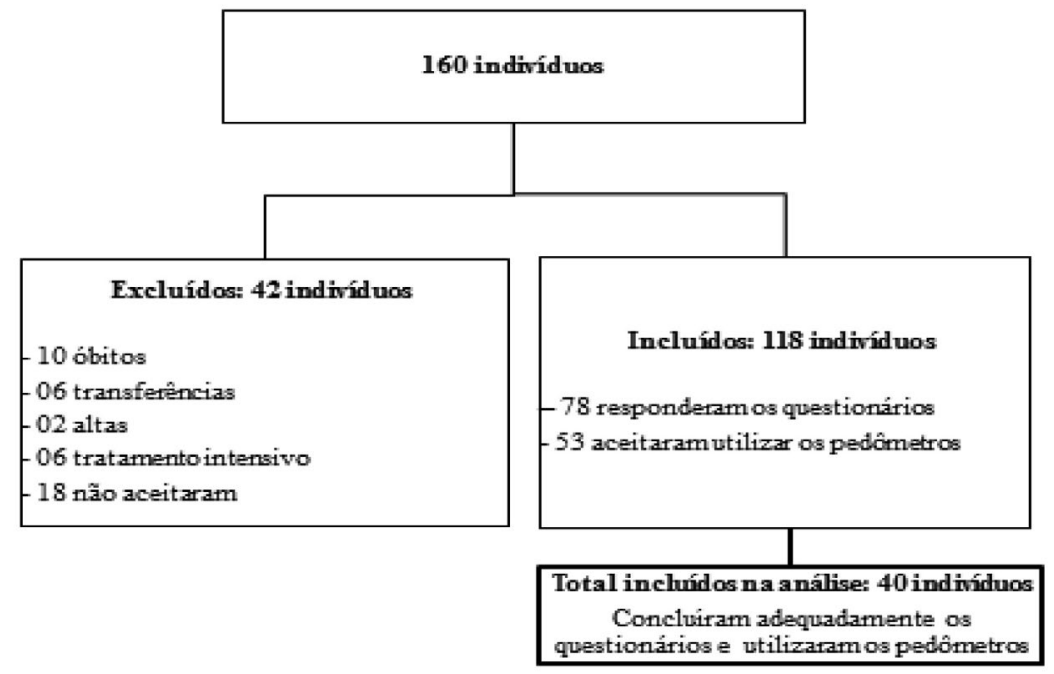

Figura 2. Dados do número de passos/dia nos pacientes renais crônicos em tratamento hemodialítico

\section{Passos/dia}

12000
10000
8000
6000
4000
2000
0


Tabela 1. Características demográficas e clínicas dos pacientes renais crônicos em tratamento hemodialítico, ( $n=40$ ). Santo Ângelo, RS - Brasil (2018/2019)

\begin{tabular}{ll}
\hline Características & Total $(\mathrm{n}=40)$ \\
\hline Idade (anos) (média $\pm \mathrm{DP})$ & $59,9 \pm 13,0$ \\
IMC (Kg/m²) (média $\pm \mathrm{DP})$ & $26,7 \pm 4,9$ \\
Tempo de hemodiálise (meses) mediana(II) & $34,5(14,2-54,7)$ \\
Sexo masculino, $\mathrm{n}(\%)$ & $28(70,0)$ \\
Etiologia da DRC, $\mathrm{n}(\%)$ & \\
HAS & $12(30,0)$ \\
DM & $6(15,0)$ \\
HAS + DM & $13(32,5)$ \\
Rins policísticos & $1(2,5)$ \\
Não especificada & $3(7,5)$ \\
Outra & $5(12,5)$ \\
Tipo de acesso, $\mathrm{n}$ (\%) & \\
Fístula AV & $28(70,0)$ \\
Cateter de longa permanência & $12(30,0)$ \\
Acompanhamento pré-dialítico, n(\%) & $24(60,0)$ \\
No passos/dia & $2690,0(918,0-5009,0)$ \\
\hline IMC: Índice de massa corporal; DRC: Doença renal crônica; AV: Arteriovenosa; Dados apresentados em média \pm desvio \\
padrão ou mediana (intervalo interquartil), conforme apropriado; II: Intervalo Interquartil.
\end{tabular}


Tabela 2. Correlação entre o número de passos/dia e a qualidade de vida pelo questionário KDQOL-SF de pacientes renais crônicos em tratamento hemodialítico. Santo Ângelo - Brasil (2018/2019)

\begin{tabular}{|c|c|c|}
\hline \multirow[t]{2}{*}{ Qualidade de Vida } & \multicolumn{2}{|c|}{ Número de passos/ dia } \\
\hline & $\mathbf{r}$ & $p$ \\
\hline Sintomas e problemas & 0,523 & $0,003^{*}$ \\
\hline Efeitos da doença & 0,458 & $0,010^{*}$ \\
\hline Sobrecarga da doença & 0,332 & 0,068 \\
\hline Situação de trabalho & 0,287 & 0,117 \\
\hline Função cognitiva & 0,323 & 0,077 \\
\hline Interação social & 0,227 & 0,219 \\
\hline Função sexual & 0,361 & $0,050^{*}$ \\
\hline Sono & 0,357 & $0,049 *$ \\
\hline Apoio social & 0,037 & 0,843 \\
\hline Encorajamento da equipe de saúde & 0,138 & 0,458 \\
\hline Saúde no geral & 0,170 & 0,362 \\
\hline Satisfação do paciente & $-0,321$ & 0,079 \\
\hline Função física & 0,617 & $<0,001$ * \\
\hline Papel físico & 0,504 & $0,004^{*}$ \\
\hline Dor & 0,496 & $0,005^{*}$ \\
\hline Estado geral de saúde & 0,297 & 0,105 \\
\hline Bem-estar emocional & 0,407 & $0,023^{*}$ \\
\hline Papel emocional & 0,435 & $0,014^{*}$ \\
\hline Função social & 0,522 & $0,003^{*}$ \\
\hline Energia/fadiga & 0,436 & $0,014^{*}$ \\
\hline Composição física & 0,598 & $<0,001$ * \\
\hline Composição mental & 0,346 & 0,057 \\
\hline
\end{tabular}

* significativo para $\mathrm{p}<0,05$.

Tabela 3. Análise de regressão linear para número de passos/dia e variáveis de perfil da amostra. Santo Ângelo - Brasil (2018/2019)

\begin{tabular}{llll}
\hline \multirow{2}{*}{ Variáveis } & Univariada & & \\
\cline { 2 - 4 } & $\mathrm{B}$ & $\mathrm{P}$ & $\mathrm{IC} 95 \%$ \\
\hline Idade & $-14,1$ & 0,788 & $-120,9-92,6$ \\
IMC & $-156,6$ & 0,262 & $-437,7-124,4$ \\
Tempo de hemodiálise & $-43,6$ & 0,089 & $-94,3-7,1$ \\
Sexo feminino & $-1513,5$ & 0,305 & $-4476,6-1449,5$ \\
Escore Saúde Geral - KDQOL-SF & 32,7 & 0,245 & $-23,7-89,2$ \\
\hline
\end{tabular}

IMC: Índice de massa corporal; KDQOL-SF ${ }^{T M}$ : Kidney Disease and Quality of Live-Short Form; $\beta$ : Coeficiente do modelo; IC: intervalo de confiança. 
Tabela 4. Qualidade de vida pelo questionário KDQOL-SFTM dos pacientes renais crônicos em tratamento hemodialítico. Santo Ângelo - Brasil (2018/2019)

$\begin{array}{ll}K D Q O L-S F^{T M} & \text { Total }(\mathrm{n}=40)\end{array}$

\section{Dimensões específicas da DRC}

1. Sintomas e problemas

$79,4 \pm 15,2$

2. Efeitos da doença

$81,4 \pm 16,8$

3. Sobrecarga da doença

$53,1(25,0-67,2)$

4. Situação de trabalho

$0,0(0,0-50,0)$

5. Função cognitiva

$93,3 \pm 9,8$

6. Interação social

$86,5 \pm 15,1$

7. Função sexual

$25,0(0,0-75,0)$

8. Sono

$74,4 \pm 17,6$

9. Apoio social

$93,7 \pm 14,9$

10. Encorajamento da equipe de saúde

$95,6 \pm 10,8$

11. Satisfação do paciente

$72,9 \pm 18,4$

Dimensões amplas

1. Função física

$55,0(26,2-75,0)$

2. Limitações físicas

$0,0(25,0-75,0)$

3. Dor

$74,0 \pm 23,4$

4. Estado geral de saúde

$37,7 \pm 19,6$

5. Bem-estar emocional

$72,6 \pm 23,0$

6. Limitações emocionais

$85,8 \pm 31,9$

7. Função social

$70,3 \pm 26,8$

8. Vitalidade

$61,4 \pm 22,6$

Saúde no geral

$64,5 \pm 21,1$

KDQOL-SF ${ }^{T M}$ : Kidney Disease and Quality of Live-Short Form; Dados apresentados em média \pm desvio padrão ou mediana (intervalo interquartil), conforme apropriado. 


\section{Discussão}

Este estudo analisou a correlação entre a atividade física e qualidade de vida, além de correlacionar o número de passos/dia com as variáveis de perfil da amostra (idade, índice de massa corporal, tempo de hemodiálise e sexo) em pacientes com doença renal em tratamento hemodialítico. Este estudo mostrou que os DRC apresentaram ser insuficientemente ativos ${ }^{11}$, com correlação positiva entre o nível de atividade física e a maioria das dimensões da qualidade de vida avaliado pelo instrumento KDQOL-SF ${ }^{\mathrm{TM}}$. Secundariamente, foi observado uma quantidade de passos insuficiente para uma vida fisicamente ativa ${ }^{11}$, assim como os menores escores de QV foram identificados nas dimensões situação de trabalho, função sexual e limitações físicas, enquanto que os maiores escores foram obtidos nas dimensões função cognitiva, apoio social e encorajamento da equipe de saúde.

A mediana do número de passos do estudo foi de 2690,0 passos. Reconhecendo que indivíduos idosos e aqueles com deficiências ou portadores de uma doença crônica são limitados em suas atividades cotidianas. Tudor-Locke ${ }^{11}$ apresentou um número de aproximadamente 5.500 passos diários ou 4.600 passos/dia em média semanal para a classificação de comportamento de vida não sedentário nesta população. Contudo, mesmo nesta consideração, a amostra apresentou-se, na grande maioria, com uma quantidade de passos insuficiente para uma vida fisicamente ativa.

O baixo nível de atividade física em DRC demonstrado no estudo foi também descrito por outros pesquisadores já há muitos anos ${ }^{12}$ e se mantém nos resultados de pesquisas mais atuais $\frac{13}{13}$ o que mantém os pacientes classificados como, na maioria dos casos, indivíduos sedentários e com baixa capacidade funcional12,13. A própria doença renal e o tratamento dialítico (inevitável) favorecem ao sedentarismo e a redução do condicionamento físico, fatores que afetam a qualidade de vida ${ }^{13}$ e a dependência funcional ${ }^{14}$, ocasionando o que conhece-se como "efeitos em cascata". Claramente, a prática regular de atividade física pode contribuir para uma melhor percepção da qualidade de vida relacionada à saúde de pacientes em hemodiálise, com benefícios em todas as dimensões ${ }^{15}$.
O exercício físico tem sido visto como um importante aliado, um coadjuvante fundamental no tratamento da DRC, associado à melhora dos parâmetros físicos e mentais ${ }^{16}$. Johansen et al. ${ }^{16}$ citam algumas variáveis encontradas que foram associadas com o baixo nível de atividade física, tais como idade avançada, sexo feminino, DM, doença aterosclerótica e baixo nível de escolaridade. Porém, nas correlações propostas neste estudo, o número de passos por dia não teve interdependência. A única variável, mais próxima da significância, foi o tempo de hemodiálise (Tabela 3). Cunha et al. ${ }^{17}$ realizaram um estudo com o objetivo de analisar a influência do tempo de hemodiálise sobre a capacidade funcional e concluíram que indivíduos com tempo de hemodiálise superior a 48 meses possuíam menor capacidade funcional, quando comparados com indivíduos em menor tempo de tratamento, o que culmina também, segundo Gomes et al. $\frac{18}{,}$ no baixo nível de atividade física.

Isoladamente, na avaliação de QV, as dimensões físicas (limitações físicas), situação de trabalho e função sexual, foram as que apresentaram os menores escores o que, consequentemente, faz-se atribuir menores escores na avaliação da saúde geral dos indivíduos. O componente físico está frequentemente relatado como importante dimensão relacionada aos baixos escores de qualidade de vida ${ }^{19}$.

Referente à situação de trabalho, sugere-se que a complexidade da doença, a condição funcional diminuída, o próprio processo de envelhecimento, além do fato de que o tratamento dialítico demanda tempo, podem ser fatores que dificultem, expressivamente, para que os indivíduos, aposentados ou não, exerçam outros trabalhos, o que reflete no baixo escore desse item da QV.

Contudo, destaca-se que a maioria das dimensões do questionário obtiveram um valor médio superior a 60 , o que enquadraria como uma boa qualidade de vida ${ }^{20}$. Os maiores escores foram obtidos nas dimensões função cognitiva, apoio social e encorajamento da equipe de saúde. Semelhantemente, Grasselli et al. 20 encontraram boas pontuações nos itens sociais e de apoio da equipe assistencial demonstrando a influência das relações pessoais nas questões de saúde. Confrontando com os presentes resultados da dimensão cognitiva, pesquisas demonstram que 
quanto maior for a gravidade da doença, maior a progressão da perda cognitiva 21 . Menciona-se, então, a importância das ações educativas para o autocuidado e para a garantia da independência nas atividades de vida diária.

Comumente, indivíduos com doença renal apresentam menores escores de QV quando comparados com a população geral. Além disso, baixas pontuações nas dimensões físicas da avaliação da qualidade de vida relacionada à saúde podem ser consideradas preditores de óbito ${ }^{22}$. É pertinente ressaltar que as queixas somáticas próprias da DRC podem mimetizar sintomas de outras doenças que também acabam interferindo em todos os domínios da QV, especialmente a depressão $0^{23}$.

Verificou-se também a alta prevalência de HAS e DM como diagnóstico etiológico da DRC. Esses dados corroboram com o Censo de Diálise Brasileira² em que 291 unidades renais responderam o inquérito e constatou-se que $58 \%$ dos indivíduos dialisados no Brasil são do sexo masculino e um percentual de $65,1 \%$ dos indivíduos se enquadram entre as faixas etárias de 45 a 74 anos. 0 mesmo censo, semelhante aos censos anteriores, demonstra a superioridade destas doenças como a etiologia da DRC. O que difere da predominância na amostra em comparação com o censo brasileiro é o índice de massa corporal. A população em diálise brasileira apresenta-se, em média, a maior parte enquadrada nos valores considerados normais/eutróficos (18,5 - 24,9), porém, a amostra apresenta valores maiores $(26,7 \pm 4,9)$, o que pode estar mostrando características mais regionais da amostra.

Embora a relação entre a melhora da qualidade de vida e bom nível de atividade física já tenha sido descrita em outros estudos com pacientes em hemodiálise ${ }^{5-7}$, este estudo traz um diferencial de mostrar cientificamente este resultado com o equipamento pedômetro que é de fácil acesso aos serviços de hemodiálise em seu processo de avaliação e acompanhamento de seus pacientes. Algumas limitações do estudo podem ser destacadas, e entre elas a avaliação de único centro regional, o que pode diminuir o poder de generalização dos resultados. Reforça-se que o perfil de características gerais da população deste estudo é similar a maioria da literatura.

\section{Conclusão}

Houve correlação positiva entre atividade física e qualidade de vida, ou seja, quanto maior a média de número de passos/dia, melhor a qualidade de vida de indivíduos em hemodiálise.

\section{Contribuições dos autores}

Cecconello $\mathrm{L}$ participou da concepção, delineamento, busca e análise estatística dos dados da pesquisa, interpretação dos resultados, redação do artigo científico, revisão e aprovação final do artigo. Morais EM participou da coleta de dados da pesquisa, revisão e aprovação final do artigo. Scopel KRO participou da concepção, delineamento e revisão e aprovação final do artigo. Stumm EMF participou revisão e aprovação final do artigo. Moreira PR participou da concepção, delineamento, interpretação dos dados, redação, revisão e aprovação final do artigo. Winkelmann ER participou da concepção, delineamento, análise estatística dos dados da pesquisa, interpretação dos resultados, redação, revisão, aprovação final do artigo.

\section{Conflitos de interesses}

Nenhum conflito financeiro, legal ou político envolvendo terceiros (governo, empresas e fundações privadas, etc.) foi declarado para nenhum aspecto do trabalho submetido (incluindo, mas não se limitando a subvenções e financiamentos, participação em conselho consultivo, desenho de estudo, preparação de manuscrito, análise estatística, etc.).

\section{Referências}

1. Bastos MG, Kirsztajn GM. Doença renal crônica: importância do diagnóstico precoce, encaminhamento imediato e abordagem interdisciplinar estruturada para melhora do desfecho em pacientes ainda não submetidos à diálise. J Bras Nefrol. 2011;33(1):93-108. https://doi.org/10.1590/S0101$\underline{28002011000100013}$

2. Sociedade Brasileira de Nefrologia - SBN. Censo de diálise 2017 [Internet]. São Paulo; 2019 (citado em 2019 mar. 20). Disponível em: http://www.sbn.org.br/censo-brasileiro

3. Medeiros NRC, Souza MNAS, Nunes RMV, Costa TS, Moraes JC, Diniz MB. Qualidade de vida relacionada à saúde de indivíduos em hemodiálise. Rev enferm UFPE on line [Internet]. 2015;9(supl.9):1018-27. Disponível em: https://periodicos.ufpe.br/ revistas/revistaenfermagem/article/viewFile/10801/11968 
4. Silva AS, Silveira RS, Fernandes GF, Lunardi VL, Backes VM. Percepções e mudanças na qualidade de vida de pacientes submetidos à hemodiálise. Rev Bras Enferm. 2011;64(5):839-44. https://doi.org/10.1590/S0034-71672011000500006

5. Fukushima RLM, Micali PN, Carmo EGD, Orlandi FDS, Costa JLR. Habilidades cognitivas e atividade física em pacientes com doença renal crônica em hemodiálise. Dement Neuropsychol. 2019;13(3):329-34. https://doi.org/10.1590/198057642018dn13-030010

6. Pei G, Tang Y, Tan L, Tan J, Ge L, Qin W. Aerobic exercise in adults with chronic kidney disease (CKD): a meta-analysis. Int Urol Nephrol. 2019;51(10):1787-95. https://doi.org/10.1007/s11255019-02234-x

7. Tsai YC, Chen HM, Hsiao SM, Chen CS, Lin MY, Chiu YW, et al. Association of physical activity with cardiovascular and renal outcomes and quality of life in chronic kidney disease. PloS One. 2017;12(8):e0183642. https://doi.org/10.1371/journal. pone. 0183642

8. Lessa LH, Granja KSB, Lira JLF, Exel AL, Calles ACN, Barbosa EA, et al. Nível de atividade física de pacientes renais crônicos submetidos à hemodiálise. ConScientiae Saúde. 2018;17(3):281-5. https://doi.org/10.5585/conssaude.v17n3.8272

9. Duarte PS, Miyazaki MCOS, Ciconelli RM, Sesso R. Tradução e adaptação cultural do instrumento de avaliação de qualidade de vida para pacientes renais crônicos (KDQOL- SFTM). Rev Assoc Med Bras. 2003;49(4):375-81. https://doi.org/10.1590/S010442302003000400027

10. Tudor-Locke C, Hatano Y, Pangrazi RP, Kang M. Revisiting “how many steps are enough?". Med Sci Sports Exerc. 2008;40(7):53743. https://doi.org/10.1249/MSS.0b013e31817c7133

11. Tudor-Locke C, Craig CL, Aoyagi Y, Bell RC, Croteau KA, Bourdeaudhuij I, et al. How many steps/day are enough? For older adults and special populations. Int. J. Behav. Nutr. Phys. Act. 2011;8(1):80. https://doi.org/10.1186/1479-5868-8-80

12. Johansen KL, Kaysen GA, Young BS, Hung AM, Silva M, Chertow GM. Longitudinal study of nutritional status, body composition, and physical function in hemodialysis patients. Am J Clin Nutr. 2003;1(4):842-6. https://doi.org/10.1093/ajcn/77.4.842

13. Medina LAR, Vanderlei FM, Vanderlei LCM, Torres DB, Padulla SAT, Freitas CEA, et al. Atividade física e qualidade de vida em pacientes com doença renal crônica submetidos à hemodiálise. ConScientia Saúde. 2010;9(2):212-9. http://dx.doi.org/10.5585/ conssaude.v9i2.2308
14. Liu CK, Lyass A, Massaro JM, D'Agostino RB, Fox CS, Murabito JM. Chronic kidney disease defined by cystatin C predicts mobility disability and changes in gait speed: The Framingham Offspring Study. J Gerontol A Biol Sci Med Sci. 2014;69(3):301-7. https://doi. org/10.1093/gerona/glt096

15. Fukushima RLM, Costa JLR, Souza OF. Atividade física e a qualidade de vida de pacientes com doença renal crônica em hemodiálise. Fisioter. Pesqui. 2018;25(3):338-44. http://dx.doi. org/10.1590/1809-2950/18021425032018

16. Johansen KL, Chertow GM, Kutner NG, Dalrymple LS, Grimes BA, Kaysen GA. Low level of self-reported physical activity in ambulatory patients new to dialysis. Kidney Int 2010;78(11):116470. https://doi.org/10.1038/ki.2010.312

17. Cunha MS, Andrade V, Guedes CA, Meneghetti CHZ, Aguiar AP, Cardoso AL. Avaliação da capacidade funcional e da qualidade de vida em pacientes renais crônicos submetidos a tratamento hemodialítico. Fisioter. Pesqui. 2009;16(2):155-60. http://dx.doi. org/10.1590/S1809-29502009000200011

18. Gomes EP, Reboredo MM, Carvalho EV, Teixeira DR, Carvalho LFCO, Ferreira Filho GF, et al. Physical activity in hemodialysis patients measured by triaxial accelerometer. Biomed Res Int. 2015;2015:645645. https://doi.org/10.1155/2015/645645

19. Fassbinder TRC, Winkelmann ER, Schneider J, Wendland J, Oliveira OB. Capacidade funcional e qualidade de vida de pacientes com doença renal crônica pré-dialítica e em hemodiálise - Um estudo transversal. J Bras Nefrol. 2015;37(1):4754. http://dx.doi.org/10.5935/0101-2800.20150008

20. Grasselli CSM, Chaves ECL, Simão TP, Botelho PB, Silva RR. Avaliação da qualidade de vida dos pacientes submetidos à hemodiálise. Rev Bras Clin Med [Internet]. 2012;10(6):503-7. Disponível em: http://files.bvs.br/upload/S/1679-1010/2012/ v10n6/a3185.pdf

21. Tamura MK, Unruh ML, Nissenson AR, Larive B, Eggers PW, Gassman J, et al. Effect of more frequent hemodialysis on cognitive function in the frequent hemodialysis network trials. Am J Kidney Dis. 2013;61(2):228-37. https://doi.org/10.1053/j. ajkd.2012.09.009

22. Oliveira MP, Kusumota L, Haas VJ, Ribeiro RCHM, Marques $\mathrm{S}$, Oller GASAO. Qualidade de vida relacionada à saúde como preditor de óbito de pacientes em diálise peritoneal. Rev LatinoAm. Enfermagem. 2016;24:e2687. https://doi.org/10.1590/15188345.0786 .2687

23. Yeh CY, Chen CK, Hsu HJ, Wu IW, Sun CY, Chou CC, et al. Prescription of psychotropic drugs in patients with chronic renal failure on hemodialysis. Ren Fail. 2014;36(10):1545-9. https://doi. org/10.3109/0886022x.2014.949762 